\title{
Determinants of business continuity management in hospitals
}

\author{
Eilia Jafar and Udita Taneja* \\ University School of Management Studies, \\ GGS Indraprastha University, \\ Dwarka, New Delhi 110078, India \\ Email: eilia.jafar@gmail.com \\ Email: udita.taneja@gmail.com \\ *Corresponding author
}

\begin{abstract}
A large number of hospitals and health facilities in India are at a high risk of being damaged by disasters. Continuity of healthcare during disasters is critical. This research surveys the hospitals in National Capital Territory of Delhi having more than 100 beds with an effort to understand and identify the determinants of business continuity management (BCM) in hospitals. The results of this study show that the six identified determinants; 1) funds for business continuity planning (BCP); 2) skills for BCM; 3) risk awareness; 4) contingency plan; 5) protocols for $\mathrm{BCM}$; 6) government schemes; are the key determinants for BCM in hospitals. Besides these, another two determinants have emerged through a qualitative survey - the mandate by government for BCP in hospitals, and protocols for business impact analysis, that need to be validated by further research.
\end{abstract}

Keywords: business continuity management; $\mathrm{BCM}$; hospital safety; disaster preparedness; disasters in hospitals; hospital contingency plan; determinants of BCM.

Reference to this paper should be made as follows: Jafar, E. and Taneja, U. (2017) 'Determinants of business continuity management in hospitals', Int. J. Critical Infrastructures, Vol. 13, No. 1, pp.57-69.

Biographical notes: Eilia Jafar is a Research Scholar at GGSIP University. She is a humanitarian professional with more than 14 years of experience in the field of disaster preparedness and response. She currently heads the Disaster Management Unit of CARE India. Her experience includes many international missions. She completed her MBA in Marketing and BTech in Civil Engineering from Aligarh Muslim University, India.

Udita Taneja obtained her BTech degree from the Indian Institute of Technology, Delhi. She pursued her MS and PhD from Tulane University, USA. This was followed by a post-doctoral fellowship at the Mayo Clinic, USA. On her return to India, she obtained her MBA degree from IIT, Delhi. She has teaching, research and industry experience of 23 years both in India and the USA. Her corporate experience in India includes having worked in the IT industry developing healthcare applications. Her current areas of research are information systems, healthcare management and management of technology. 


\section{Introduction}

Business continuity management (BCM) is the development and implementation of a step-by-step plan for how any business establishment will recover and restore, either partially or completely, interrupted critical functions within a predetermined time after a disaster or an extended disruption.

Despite the fact that growing economies like India are proud providers of the best possible healthcare at an affordable price, governments investment in this varies significantly from country to country. These critical facilities are at a high risk of collapse in case of any untoward incident or a disaster. The poor performances of health facilities during and in the aftermath of a disaster are often attributed to the lack of funds by health administrators. There is a need to identify the factors that contribute to better performance of health facilities in emergencies by virtue of business continuity planning and implementation of such plans.

\section{Study background}

A determinant is defined as an element that identifies or determines the nature of something or that fixes or conditions an outcome (Merriam-Webster, 2015). Based on the prediction of various emergency situations, "a Business Continuity Plan (BCP) determines what activities need to be performed to prepare for a disaster. It also includes the methods and means for ensuring continuity of business in an emergency to minimize damage to business property and to facilitate continuation of core business and early recovery" (Kudo et al., 2013).

Business continuity planning looks at various domains and elements in a business and prepares for their continuity post a disaster within a stipulated timeframe. This also involves strengthening of certain systems and processes, some of which require ear-marked human and financial resources. Following the September 11, 2001 attack in the USA, there was an increase in funding for preparedness in hospitals. The funding could be utilised for developing and implementation of the preparedness plan, monitoring of diseases, improved communication and training of hospital staff. A study was done to examine the impact of the added responsibilities on the authorities due to additional fund allocation.

Three recommendations were made:

1 funding for public health professionals should be increased

2 increase training and capacity building of staff

3 increase coordination and communication between different stakeholders in emergency planning (Hyde et al., 2006). 
There is a strong linkage between business continuity and financial sustainability. While business continuity looks at a plan to recover and maintain a business during a disaster, financial sustainability looks at the economic viability of an organisation during and post a disaster event. For business continuity planning the existing laws and regulations regarding claims, waivers and sources of income should be considered for a hospital to continue to provide healthcare services (ASPR, 2015).

Development and implementation of business continuity processes requires certain set of skills. According to Zawada (2011), there are ten attributes that must be demonstrated by business continuity professionals:

1 ability to communicate

2 ability to work with both business and technology professionals

3 ability to think in terms of processes

4 ability to analyse

5 ability to lead, facilitate and influence

6 ability to stay organised

7 ability to sell and motivate

8 ability to objectively perform cost-benefit analyses and make unbiased recommendations

9 ability to be creative, while using proven business continuity planning processes

10 ability to learn and understand existing and emerging management practices.

The methodology for development of a business continuity plan comprises of setting up a strong committee for governance that is aware of the key decisions it needs to take; the results of the business continuity plan; and is aware of the risks. During the mitigation phase of emergency management in hospitals, understanding of risks (risk assessment) and business impact analysis (BIA) are the two key components of business continuity (Robles et al., 2012).

Developing a comprehensive emergency preparedness plan includes risk analysis to foresee the potential threats and plan for them. Some hospitals may have the expertise to plan and conduct drills for testing the disaster preparedness plan but many lack such skills (Hick et al., 2012).

\subsection{Availability of funds}

The funding for public health facilities in India is limited. This adversely impacts trauma care in cases where the services are not based on payment of fee (Joshipura et al., 2003). Hospitals require huge front end investment and facilities that are unable to raise sufficient funds are unable to provide adequate healthcare (Berhie, 1991). There is an ongoing debate on the impact of funding for emergency disaster preparedness in health facilities on the health of communities (Hyde et al., 2006). 


\subsection{Skills}

Disaster training of healthcare providers is an important aspect of disaster preparedness to improve knowledge and skills of healthcare providers (Williams et al., 2008). Public health practice can be improved by enhancing the skills to respond in different disaster scenarios (Hoard et al., 2005).

\subsection{Risk awareness}

It is important to increase risk awareness for earthquake preparedness. In a study conducted in Japan post the Great East Japan Earthquake, furniture anchoring for securing non-structural elements was found to be associated with risk awareness (Haraoka et al., 2013).

\subsection{Contingency plans}

Many hospitals in India have mass casualty plans or business contingency plans. Such plans look at some of the aspects of business continuity and are definite steps towards business continuity planning. These plans mainly focus on disaster response actions and do take into consideration the potential hazards.

\subsection{Standard protocols for BCM}

Government schemes to fund and promote business continuity plans in hospitals have played a significant role in improving the status of emergency preparedness in hospitals. Centers for Disease Control and Prevention (CDC) and its Office of Public Health Preparedness and Response (PHPR), (the CDC is a part of the Department of Health and Human Services in the USA and works to protect the country from health and safety security threats), invest in public health to improve the status of preparedness in hospitals at various levels. Funding is done through cooperative agreements. In the absence of such funding and support for response capacity development, many hospitals had low levels of preparedness, e.g., Republic of Palau with the support from CDC has been able to make a big leap from a status of 'no documented plan' to a department that has the capacity to activate an emergency operation centre within one hour of an emergency (PHPR, 2015). "The business impact analysis (BIA) and risk assessment are foundational elements of every effective business continuity program" (Rupert, 2015).

\subsection{Government schemes}

Under Hyogo framework for Action 2, integration of health and establishment of community health resilience are critical. It is recommended that greater government incentives should be provided where there is low health resilience (Otomo and Burkle, 2014). Government should develop schemes and policies that support public health preparedness. 


\subsection{Conceptual framework}

Based on the literature review, a conceptual framework was developed. Six determinants of BCM in hospitals were identified as follows:

Determinants:

1 availability of funds

2 skills for BCM in hospitals

3 risk awareness

4 existence of a hospital disaster contingency plan

5 standard protocols for BCM

6 government schemes to fund and promote business continuity plans in hospitals.

Figure 1 Model for determinants of BCM (see online version for colours)

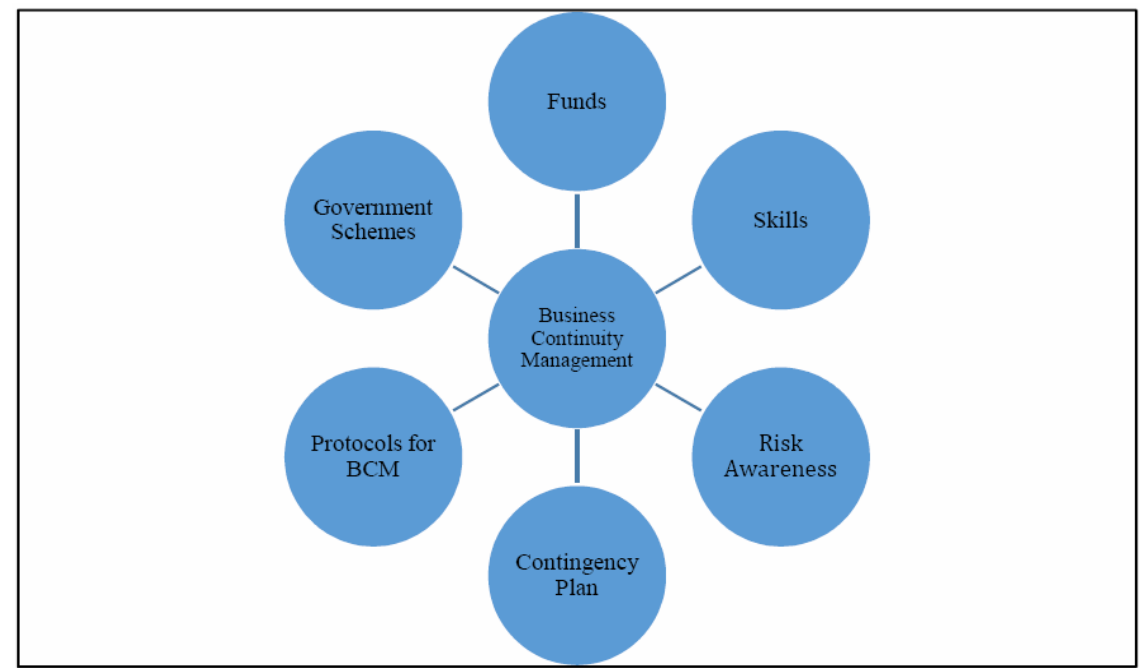

This concept presents the six key determinants of BCM.

\section{Research methodology}

A concurrent mixed-methods research design was applied by developing a survey questionnaire with both qualitative and quantitative questions. Data related to both the quantitative and qualitative phases of the study were collected simultaneously. 
Based on an exhaustive literature review, a detailed interview schedule was developed to identify the critical determinants for hospital continuity planning as perceived by health professionals and to understand the deep-rooted underlying causes for poor and non-existent business continuity plans in health sector. The questionnaire was shown to academic and health experts to check for its relevancy and flow, i.e., content validity. The modified questionnaire was then used for conducting personal interviews.

Inclusion criteria: All the hospitals with more than 100 beds were approached. Out of 85 such hospitals, 63 hospitals responded positively to the request for interviews. In each hospital one interview was conducted with the focal person responsible for safety in the hospital, and the other 1-2 interviewees were selected based on nomination from the focal person. 217 interviews were successfully conducted with 190 doctors and officials (human resource department/administration department/safety and security officer) from 63 private and government hospitals. In addition to above, a list of 40 experts from government, academics and civil society organisations in Delhi was drawn and the experts were approached for key informant interviews. Based on positive responses, 27 experts working in the field of health and disaster management (DM) were personally interviewed.

Ethical considerations: informed consent was obtained from each of the study participants before conducting the interviews. The study did not involve any other ethical clearance. The responses obtained were then analysed with the help of SPSS-21, a statistical package. Descriptive statistics were used to summarise the data with frequencies and crosstabs procedures.

\section{Data analysis}

Based on the literature review for determinants of BCM, six determinants were identified and officials from hospitals as well as public health experts were interviewed to ascertain whether the identified determinants were the key determinants and also to understand if there was any determinant that was left out. As the interview was semi-structured, it also allowed the respondents to add determinants, other than those listed. The following data analysis shows the profile of respondents, the ratio of government and private hospitals, and the analysis of determinants. In order to establish that that the identified determinants are the key determinants a cut-off value has been proposed. Determinants for which more than $2 / 3 \mathrm{rd}(66.6 \%)$ of the respondents agree that it is a key determinant will be confirmed as the key determinants. This will include the 'strongly agree' as well as 'agree' categories. This implies that if more than 1/3rd (33.3\%) of the respondents 'strongly disagree'; 'agree' or do not know/not sure, we will not consider it as a key determinant.

\subsection{Determinants}

Once the BIA has been done in a business establishment for the purpose of business continuity planning, many areas are identified for action to improve preparedness for any disaster event and ensure continuity. Such actions may also require human, technical and financial resources. Tables 1 and 2 show the consolidated response from officials interviews from hospitals and health experts interviewed as a part of this research respectively. 
Table 1 Data from interviews in hospitals

\begin{tabular}{lcccccc}
\hline \multicolumn{7}{c}{ Hospital interviews data } \\
\hline Agreement & $\begin{array}{c}\text { Funds } \\
\text { availability } \\
(\%)\end{array}$ & $\begin{array}{c}\text { Skills } \\
(\%)\end{array}$ & $\begin{array}{c}\text { Risk } \\
\text { awareness } \\
(\%)\end{array}$ & $\begin{array}{c}\text { Hospital } \\
\text { contingency } \\
\text { plan (\%) }\end{array}$ & $\begin{array}{c}\text { Standard } \\
\text { protocols } \\
\text { for BCM } \\
(\%)\end{array}$ & $\begin{array}{c}\text { Government } \\
\text { schemes (\%) }\end{array}$ \\
\hline Strongly agree & 77.9 & 96.8 & 77.9 & 91.6 & 85.3 & 77.9 \\
Agree & 16.8 & 3.2 & 16.8 & 6.3 & 12.6 & 16.8 \\
Do not know & 0.5 & 0 & 0.5 & 0.5 & 0.5 & 0.5 \\
Disagree & 4.7 & 0 & 4.7 & 1.6 & 1.6 & 4.7 \\
Strongly disagree & 0 & 0 & 0 & 0 & 0 & 0 \\
\hline
\end{tabular}

As per the respondents from hospitals, more than $90 \%$ of respondents strongly agree on 'Skills' and 'Contingency planning' as the key determinants of BCM. This is followed by $85 \%$ respondents strongly agreeing to standard protocols for BCM. $77.9 \%$ of respondents from hospitals strongly agreed that funds availability, risk awareness and government schemes are key determinants. On the remaining three determinants (availability of funds, risk awareness and government schemes) there was strong agreement by $77.9 \%$ of the respondents. There was no disagreement on skills as a key determinant, however $4.7 \%$ of the respondents from hospitals disagreed on funds availability, risk awareness, and government schemes as the key determinants of BCM. This was followed by $1.6 \%$ of the respondents disagreeing on contingency plan, and protocols for BCM as key determinants.

The interviews done with experts, however, present a slightly different picture.

Table 2 Data from interviews of experts

\begin{tabular}{lcccccc}
\hline \multicolumn{7}{c}{ Experts interview data } \\
\hline Agreement & $\begin{array}{c}\text { Funds } \\
\text { availability } \\
(\%)\end{array}$ & $\begin{array}{c}\text { Skills } \\
(\%)\end{array}$ & $\begin{array}{c}\text { Risk } \\
\text { awareness } \\
(\%)\end{array}$ & $\begin{array}{c}\text { Hospital } \\
\text { contingency } \\
\text { plan (\%) }\end{array}$ & $\begin{array}{c}\text { Standard } \\
\text { protocols } \\
\text { for BCM } \\
(\%)\end{array}$ & $\begin{array}{c}\text { Government } \\
\text { schemes (\%) }\end{array}$ \\
\hline Strongly agree & 11.1 & 81.5 & 70.4 & 44.4 & 11.1 & 77.8 \\
Agree & 66.7 & 18.5 & 29.6 & 55.6 & 66.7 & 22.2 \\
Do not know & 0 & 0 & 0 & 0 & 22.2 & 0 \\
Disagree & 22.2 & 0 & 0 & 0 & 0 & 0 \\
Strongly disagree & 0 & 0 & 0 & 0 & 0 & 0 \\
\hline
\end{tabular}

More than $80 \%$ of experts interviewed strongly agreed on 'Skills' as a key determinant in BCM. This was followed by $77 \%$ of respondents strongly agreeing on 'Government schemes and policies' to promote hospital disaster preparedness and $70.4 \%$ in strong agreement of 'Risk awareness' as a key determinant.

In addition to the above, $51 \%$ of the respondents from hospitals mentioned protocols for BIA as an important determinant. 55\% of experts interviewed also mentioned protocols for BIA are a key determinant. Another determinant that a majority of experts mentioned was government's mandate. $74 \%$ of the experts interviewed mentioned this as 
a key determinant. During interviews with hospital officials this was mentioned only by $25 \%$.

As we see from both datasets, Skills is rated by the highest number of respondents as a key determinant. It is important to note that $22.2 \%$ of the experts interviewed do not see 'Funds Availability' as a key determinant. Funding is perceived as a key determinant by officials in hospitals, while very few experts see it as a significant determinant. During interview the experts shared that a lot can be done from the existing resources available in the hospitals and funding is not necessarily a barrier.

\section{Results}

Profile of respondents: Out of the 190 respondents from hospitals, 98 respondents were from government hospitals and 92 were from private hospitals. As highlighted in Figure 2, the respondents from hospitals comprised of Medical Superintendents (MS), Chief Medical Officers (CMO), Medical Officers (MO), Heads of Departments, Doctors, Safety and Security focal persons assigned by the hospital authorities or those from an outsourced security team.

Figure 2 Functions of respondents from hospitals (see online version for colours)

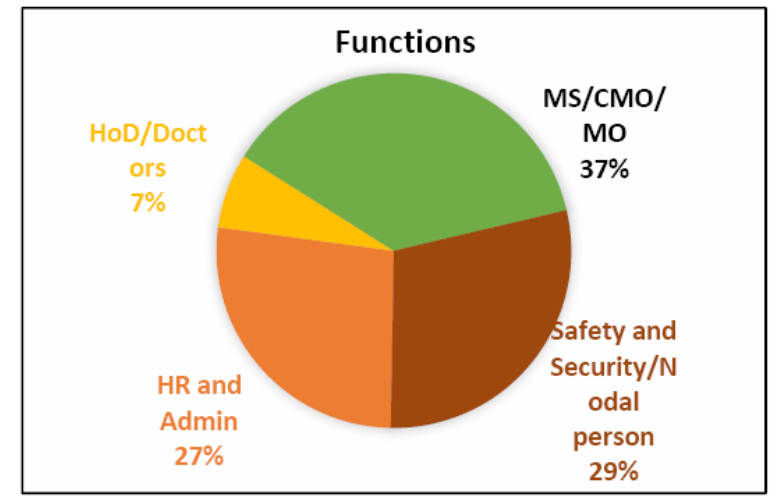

Figure 3 Sex ratio of respondents from hospitals (see online version for colours)

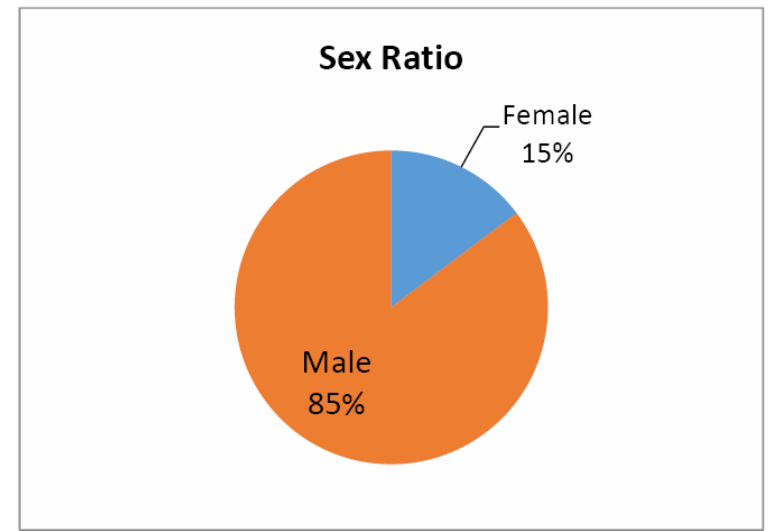


Out of the respondents from hospitals, $15 \%$ were females while the rest of the respondents were males.

Besides interviews in hospitals, interviews with 27 health experts (government officials, civil society officials, officials from multi-lateral organisations) were also done. Hence, the total numbers of interviews conducted were 217.

Table 3 Types of respondents

\begin{tabular}{lcc}
\hline Type of respondents & Number of respondents & Percent \\
\hline Health sector experts & 27 & 12.4 \\
Private hospitals & 92 & 42.4 \\
Government hospitals & 98 & 45.2 \\
Total & 217 & 100.0 \\
\hline
\end{tabular}

Four out of the $27(14.8 \%)$ experts interviewed were females.

\subsection{Themes emerging from the interviews}

During the interviews in hospitals, the respondents shared that he hospitals in Delhi are at risk to earthquakes, fire, floods, building collapse (in case of old or poorly constructed buildings), bomb attacks, heavy storms and leakage of chemical or radio-active gases. $\mathrm{BCM}$ needs to consider all these risks.

Some of the reasons quoted for poor or non-existent business continuity plans in government hospitals were the following:

1 Lack of training.

2 Lack of sincerity and willingness.

3 There is no incentive in engaging with preparedness work. It is an additional task.

4 No protocols for preparedness.

5 Over-crowding of patients.

6 Lack of accountability.

7 Lack of advanced equipment and infrastructure.

8 Less number of staff.

9 Low bed strength compared with demand.

10 Lack of specialty services.

11 Need for testing of plans by conducting drills.

12 Lack of awareness and sensitisation.

13 Lack of funds for addressing the gaps in preparedness.

14 Lack of access to modern technology.

15 Lack of coordination between various departments. 
The following reasons were quoted by the officials of private hospitals for poor or non-existent business continuity plans in some hospitals:

1 Lack of trained manpower.

2 Lack of time, extremely busy schedules do not allow hospital officials to give time for preparedness planning.

3 Focus on meeting the requirements of accreditation.

4 Lack of involvement of staff in preparedness planning. Plans are developed by focal persons but dissemination with proper understanding is limited.

5 Lack of seriousness towards disaster preparedness.

6 Lack of funds for the purpose of preparedness, mitigation and retrofitting interventions.

7 Taking decisions is easy in private hospitals but in government hospitals every approval follows a long process.

8 Lack of protocols in place.

9 Lack of coordination between hospitals, especially at the time of referring to another hospital it is not checked whether the other hospital has the capacity to treat the patient.

\subsection{Agreement on the proposed model}

All the six determinants in the proposed model have been found by the survey respondents as the key determinants for BCM. If we look at all the determinants together we see that the agreement for determinants (agree to strongly agree) ranges from $81.5 \%$ to $98.15 \%$.

Figure 4 Agreement on the proposed model (see online version for colours)

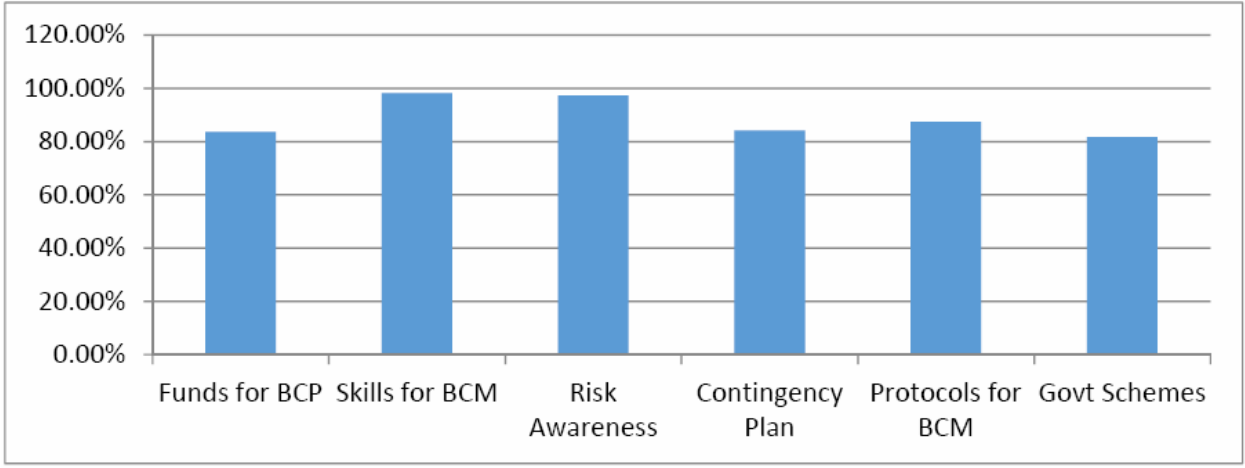




\section{Discussion}

There is highest level of agreement for 'Skills' as a key determinant that has emerged from interviews of both the officials from hospitals but also during the interviews of the sector experts. Unless people understand the risks they are exposed to, they will not be able to plan to deal with it. Ignorance is one of the biggest reasons for lack of preparedness. This is the reason why there is also a strong agreement $(77.9 \%$ respondents from hospitals and 70.4\% experts) on 'Risk Awareness' as a key determinant. Once the risk is understood effective planning requires knowledge and skills. There is a need for awareness of hazard risks in hospitals in India and capacity building of officials on business continuity planning with a focus of planning in hospitals. 'Contingency Plans' were identified as a key determinant with most of the respondents saying that if hazard specific contingency plans are in place people know their responsibilities and act accordingly at the time of a hazardous event. Hospitals will be able to recover from the impact of the hazard quickly and continue to provide treatment, a key function of health facilities. 'Protocols for BCM' significantly help planners and stakeholders to act as per the response plan and were therefore identified as a key determinant. The other determinant found to be significant in case of hospitals in Delhi was 'Government schemes' to promote business continuity planning and the incentives linked to it. Both civil societies and government bodies have a role to play in hospital business continuity planning (Acosta and Chandra, 2013).

Respondents also agreed that 'Funds for BCP' are important because many areas identified for action during BIA and planning require financial resources.

Additional determinants: As a part of qualitative questions the respondents were also asked if there were any other determinants of BCM in hospitals that have not been listed. A few experts indicated that a government mandate is a key determinant. According to the CEO, Geo Hazards Society (personal interview 2014), in the absence of a mandate the plans are either non-existent or poor and ineffective. Some experts also believe that although a part of BCM, the protocols for BIA are also required because a poor analysis may result in poor planning. BIA forms a basis for business continuity planning but investment in BIAs by management remains a challenge (Rupert, 2014). The two additional determinants need to be further validated by conducting a similar study.

\section{Conclusions}

The six determinants

a funds for BCP

b skills for BCM

c risk awareness

d contingency plan

e protocols for $\mathrm{BCM}$

f government schemes are the key determinants for BCM in hospitals. 
There is a need to further investigate and establish the validity of the additional two determinants

1 government mandate

2 protocols for BIA that emerged during the interviews with health experts and officials in hospitals.

For $\mathrm{BCM}$ in hospitals in India, there is a need to engage in all the six identified determinants and also consider the additional two determinants. Accountability needs to be clearly established along with capacity building of focal persons. Time should be built in for involvement in preparedness actions as a part of key roles and responsibilities. In view of the challenges of limited understanding of plans in facilities where plans to exist, it is recommended that testing of plans through simulation of different hazard scenarios be made mandatory in hospitals. Accreditation should also be based on testing. There is a need for inter-hospital coordination and business continuity planning should take into account the offsite factors.

\section{References}

Acosta, J. and Chandra, A. (2013) 'Harnessing a community for sustainable disaster response and recovery: an operational model for integrating nongovernmental organizations', Disaster Medicine and Public Health Preparedness, Vol. 7, No. 4, pp.361-368 [online] http://www.journals.cambridge.org/abstract_S1935789312000018 (accessed 20 July 2014).

ASPR (2015) Healthcare COOP and Recovery Planning, January [online] http://www.neflhcc.org/ASPR_Healthcare_COOP_Guidance.pdf (accessed 24 January 2016).

Berhie, G. (1991) 'Emerging issues in health planning in Saudi Arabia: the effects of organization and development on the health care system', Social Science and Medicine, Vol. 33, No. 7, pp.81-24.

Haraoka, T. et al. (2013) 'Factors related to furniture anchoring: a method for reducing harm during earthquakes', Disaster Medicine and Public Health Preparedness, Vol. 7, No. 1, pp.55-64 [online] http://www.journals.cambridge.org/abstract_S1935789313000359 (accessed 10 May 2014).

Hick, J.L., Hanfling, D. and Cantrill, S.V. (2012) 'Allocating scarce resources in disasters: emergency department principles', Annals of Emergency Medicine, Vol. 59, No. 3, । pp.177-187 [online] http://dx.doi.org/10.1016/j.annemergmed.2011.06.012 (accessed 5 November 2015).

Hoard, M. et al. (2005) 'Systems modeling in support of evidence-based disaster planning for rural areas', International Journal of Hygiene and Environmental Health, Vol. 208, Nos. 1-2, pp.117-125.

Hyde, J. et al. (2006) 'Better prepared but spread too thin: the impact of emergency preparedness funding on local public health', Disaster Management and Response, Vol. 4, No. 4, pp.106-113.

Joshipura, M.K. et al. (2003) 'Trauma care systems in India', Injury, Vol. 34, No. 9, pp.686-692.

Kudo, D. et al. (2013) 'Resources for business continuity in disaster-based hospitals in the Great East Japan earthquake: survey of Miyagi prefecture disaster base hospitals and the prefectural disaster medicine headquarters', Disaster Medicine and Public Health Preparedness, Vol. 7, No. 5, pp.461-466 [online] http://www.journals.cambridge.org/abstract_S1935789313000773 (accessed 5 November 2015).

Merriam-Webster (2015) Determinant [online] http://www.merriamwebster.com/dictionary/determinant (accessed 27 November 2015). 
Office of Public Health Preparedness and Response (PHPR) (2015) National Snapshot of Public Health Preparedness, pp.4-32 [online] http://www.cdc.gov/phpr/pubslinks/ 2015/index.html (accessed 5 November 2015).

Otomo, Y. and Burkle, F.M. (2014) 'Breakout session 1 summary: frameworks and policies relating to medical preparedness and health management in disasters', Disaster Med. Public Health Prep., Vol. 8, No. 4, pp.359-360.

Robles, T. et al. (2012) Lead Toolkit Coordinators Hospital Continuity Planning Workgroup, October.

Rupert, J. (2014) 'Establishing the business case for the business impact analysis', Avalution's Perspective, March, pp.10-13.

Rupert, J. (2015) 'Introducing ISO 22317 - the business impact analysis standard', Disaster Resource Guide, pp.10-12.

Williams, J., Nocera, M. and Casteel, C. (2008) 'The effectiveness of disaster training for health care workers: a systematic review', Annals of Emergency Medicine, Vol. 52, No. 3, pp.7-9.

Zawada, B. (2011) 'Key skills a business continuity professional must have or obtain: 2011 and beyond', Avalution's Perspective, pp.10-12.
Abbreviations
BCM business continuity management
BCP business continuity plan
BIA business impact analysis
CMO chief medical officer
HR human resource
MO medical officer
MS medical superintendent. 